\title{
Optimal Spectrum Sensing Interval to Minimize theTransmission Delay for Cognitive Radio
}

\author{
Shuai $\mathrm{Li}^{1}$, Peng Gong ${ }^{1, \mathrm{a}}$, Qian Yang ${ }^{1, \mathrm{~b}}$, Mingguan $\mathrm{Li}^{1, \mathrm{c}}$, and Linchuan $\mathrm{Ma}^{2, \mathrm{~d}}$
}

${ }^{1}$ National Key Laboratory of Mechatronic Engineering and Control, School of Mechatronical Engineering, Beijing Institute of Technology, Beijing, China. Gong Peng is the corresponding author.

${ }^{2}$ Samsung Electronics Co.,Ltd. Yongin-City, Gyeonggi-Do, 446-711, South Korea.

apenggong@bit.edu.cn, byangqian@bit.edu.cn,'liming.Img@gmail.com, dmalinchuan@gmail.com

Keywords: Cognitive radio, spectrum sensing interval, delay

\begin{abstract}
In this work, we have studied the optimal spectrum sensing interval, which is the time between two consecutive spectrum sensing activities, such that the delay of the cognitive transmission is minimized. The throughput maximization of the cognitive radio is always a common goal of the researchers, which is keenly related to the delay of the cognitive transmission. Whereas the delay is mostly decided by the spectrum sensing interval for a given primary user (PU) channel. Therefore, we have studied the setting of the spectrum sensing interval to minimize the transmission delay of the secondary user (SU). Especially, the retransmission is considered, which deteriorates the delay of the cognitive transmission; however, neglected by the most studies. Finally, we provide both the optimal setting of the spectrum sensing interval and the numerical values.
\end{abstract}

\section{Introduction}

In Cognitive Radio (CR), the unlicensed data delivered is enabled on a free licensed frequency band, while the licensed transmission is protected. CR has exhibited great potential in mitigating the unbalanced utilization of the limited precious spectrum resources [1]. Generally, there are two major functions in CR, the spectrum sensing and dynamic spectrum access. The former performs the role of spectrum opportunities discovery and licensed user protection, whereas, the later conducts the data delivery. The spectrum sensing is our priority, thus let us have a brief look over it [2].

There are many literatures concerning the spectrum sensing methods, which can be generally classified as, energy detection [3], match-filter based spectrum sensing [4] and feature based spectrum sensing [5]. Energy detection decides the state of PU according to the observed energy level [3]; however, its detection performance is sensitive to the noise influence. The match-filter based spectrum sensing has the theoretically best performance, whereas the PU signal pre-knowledge is required [4]. The feature based spectrum sensing methods have certain requirements of the PU signal and conduct the sensing accordingly, e.g., in general likelihood ratio test (GLRT) based spectrum sensing [5], the distribution of the PU signal is demanded.

The spectrum sensing can be performed in the view of MAC (media access control) layer, however less studied. The up-to-date works about MAC sensing are mostly about the spectrum opportunity discovery and PU interference reduction. In [6], the efficient discovery of the spectrum opportunities is studied, while the protection of PU is not accounted. The scheduling of the spectrum sensing is investigated in [7] for a non-periodical spectrum sensing. Proactive spectrum sensing is developed to reduce the interference to PU before the collision between PU and SU occurs [8]. The order of sensing a group of PU channels is studied in [9].

In most of the aforementioned works, the relationship between spectrum sensing and delay of the SU data delivery is not considered. Since spectrum sensing is a basic function, which discovers the spectrum opportunities with overhead, it directly decides the throughput of SU. Moreover, since the throughput maximization is always a common goal of the cognitive radios and throughput is keenly related to the delay, we will study the spectrum sensing interval, i.e., the time between two consecutive sensing activities, such that the transmission delay is minimized. Especially, the 
retransmission is considered, which is neglected even by most of the literatures about DSA [10]. The optimization problem is formulated and solved. The optimal sensing interval is provided with the numerical results.

The rest of the paper are organized as follows. In section II, system model and some preliminaries are provided. In Section III, the problem of minimizing the transmission delay of SU with an optimal spectrum sensing interval is formulated and solved. The numerical results are provided in Section IV. Finally, Section V draws conclusion.

\section{System Model and Preliminaries on Primary User}

\section{System Model}

For simplicity, one PU channel is considered. Since the traffic of multiple PUs can be treated as one single PU, one PU is considered. The secondary user conducts periodical spectrum sensing, with sensing time window $t_{\text {win }}$ and spectrum sensing interval $\tau$. We consider the case that one retransmission of the incorrectly received SU data would satisfy the requirement of the application, e.g., quality of service.

A PU channel with error free spectrum sensing is considered, i.e., with zero probability of false alarm $P_{f}$ and miss alarm $P_{m}$. The erroneous case of the problem can be conceived from the error-free case. It will be explained later.

\section{Preliminaries on Primary User}

The variation of the PU states is generally modeled as an alternative renewal process. Define $Z(t)$ as this renewal process of the PU channel. $Z(t)=1$ (state “ 1 ”), if the PU channel is "on" at time $t$; otherwise when the PU channel is “off”, $Z(t)=0$ (state “ 0 ”).

$Z(t)$ is characterized by a pair of probability density functions $f^{1}(t)$ and $f^{0}(t)$, which denote the probability distribution of the sojourn duration of “on” period $T^{1}$ and "off” period $T^{0}$, respectively. $f^{1}(t)$ and $f^{0}(t)$ are considered following exponential distributions with known $\theta^{1}$ and $\theta^{0}$, as shown by Eq. 1. $E\left[T^{1}\right]=1 / \theta^{1}$ and $E\left[T^{0}\right]=1 / \theta^{0}$, where $E[\cdot]$ is the expectation operation.

$$
\left\{\begin{array}{l}
f^{1}(t)=\theta^{1} e^{-\theta^{1} t} \\
f^{0}(t)=\theta^{0} e^{-\theta^{0} t}
\end{array}\right.
$$

The relationship of two time consecutive states of PU observed by SU is described by a set of conditional probabilities

$P^{i, j}(\Delta t)$, with $i, j \in\{0,1\}, \Delta t>0$, which denotes the probability that state $j$ is now observed, if state $i$ was observed $\Delta t$ time before.

$$
P^{i, j}(\Delta t)=u^{j}(1-u)^{1-j}+(-1)^{j+1} u^{1-i}(1-u)^{i} e^{-\left(\theta^{1}+\theta^{0}\right) \Delta t}
$$

where $u=\theta^{0} /\left(\theta^{1}+\theta^{0}\right)$ is defined as channel utilization of this PU channel.

\section{Problem Formulation}

We consider the delay induced by the delivery of an SU data packet $T_{p}$ on a PU channel. $T_{p}>>\tau$ and $T_{p}$ is partitioned into subpackets with equal length $\tau$ and delivered.

Define $D\left(T_{p}, \tau\right)$ as the delay induced by delivering an SU packet $T_{p}$ on a PU channel, while the sensing is performed with interval $\tau . D\left(T_{p}, \tau\right)$ is considered consisting of two parts, i.e., $D_{b s}\left(T_{p}, \tau\right)$ and $D_{r e}\left(T_{p}, \tau\right)$. The former denotes the expected delay induced by the busy periods of PU and the spectrum sensing overhead, and the latter is the delay caused by data retransmission, e.g., in case of 
incorrect receiving. Let $T_{r e}\left(T_{p}, \tau\right)$ denote the average length of retransmitted data within $T_{p}$. The delivery of $T_{r e}\left(T_{p}, \tau\right)$ produces $D_{r e}\left(T_{p}, \tau\right)$. We intend to minimize $D\left(T_{p}, \tau\right)$ with a proper $\tau$,

\section{Derivation of $D\left(T_{p}, \tau\right)$}

$$
\tau^{*}=\arg \min _{\tau}\left\{D\left(T_{p}, \tau\right)\right\}
$$

For a PU channel with given $T_{p}$ and $\tau, D\left(T_{p}, \tau\right)$ depends on $u$ and the length of the sensing time window $t_{\text {win }}$. We have following considerations.

- Because of retransmission, the actual transmitted data length is $T_{p}+T_{r e}\left(T_{p}, \tau\right)$.

- For a given PU channel, the "off” portion of the PU channel, which can be utilized by SU, is $1-u$.

- Due to the spectrum sensing overhead (the sensing time window $t_{\text {win }}$ ), the portion of the PU channel, which can be used for SU data deliver is reduced to $(1-u) \frac{\tau}{t_{\text {win }}+\tau}$.

- During the sensing window twin, no SU data is delivered. Thus, delay is produced, which should be accounted in $D\left(T_{p}, \tau\right)$. The portion of the PU channel, which is occupied by the spectrum sensing time window during the usable part of the PU channel, is $(1-u) \frac{t_{\text {win }}}{t_{\text {win }}+\tau}$.

The busy portion of the PU channel, during which PU data is delivered, together with the sensing overhead (the last item above), are right the delay. Therefore, putting above four items together, we obtain $D\left(T_{p}, \tau\right)$ in Eq. 4,

$$
D\left(T_{p}, \tau\right)=\frac{\left(T_{p}+T_{r e}\left(T_{p}, \tau\right)\right)}{(1-u) \frac{\tau}{t_{\text {win }}+\tau}}\left(u-(1-u) \frac{t_{\text {win }}}{t_{\text {win }}+\tau}\right)=\left(T_{p}+T_{r e}\left(T_{p}, \tau\right)\right) \frac{u \tau+t_{\text {win }}}{(1-u) \tau}
$$

where $T_{r e}\left(T_{p}, \tau\right)$ is derived next, considering retransmission. Usually, when the number of erroneous received data exceeds a threshold, retransmission is initiated. The key point is the outrage probability that the erroneously delivered SU data exceeds a predefined threshold. It is studied next.
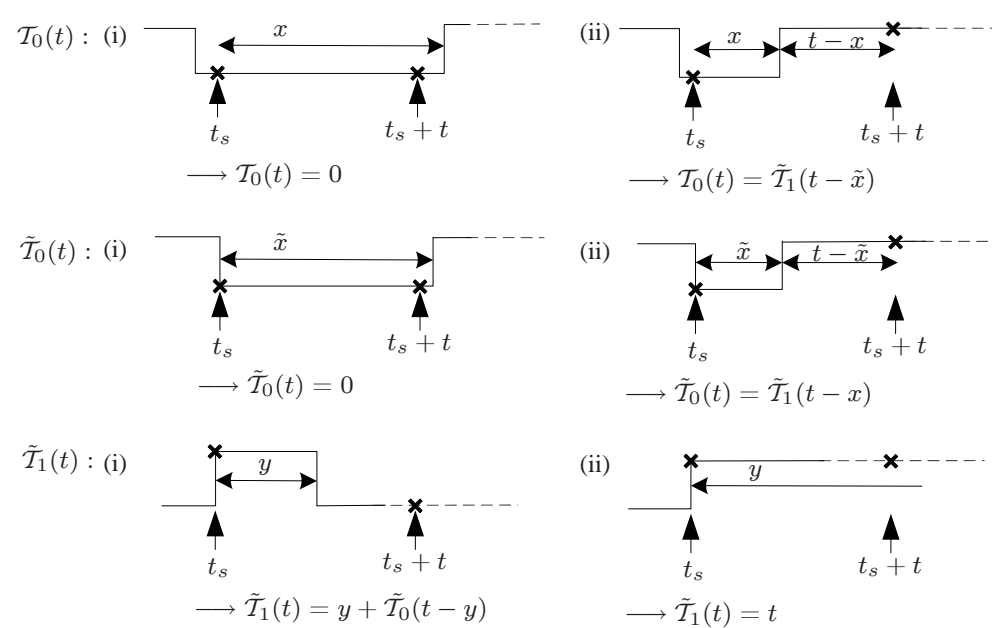

Fig. 1. The possible cases of $\tau_{d}(t)$ and $\tilde{\tau}_{d}(t),(d=0,1) . x$ indicates the remaining time in the current "off" state beginning from $t_{s} \cdot x$ is denoted as $x^{\sim}$ when state changes at $t_{s} \cdot y$ and $\tilde{y}$ indicate that for the "on" case. $\tau_{d}(t)$ and $\tilde{\tau}_{d}(t),(d=0,1)$ can be considered as the "dual" of their counterparts in [6]. This figure is plotted according to this duality. 
Table 1 Parameter Definitions for Figure 1

\begin{tabular}{|l|l|}
\hline Symbol & Definitions \\
\hline$t_{s}$ & The sampling time (sensing instant) \\
\hline$\tilde{x},(\tilde{x}>0)$ & The remaining time of staying current "on" state counting from $t_{s}$ \\
\hline$\tilde{y},(\tilde{y}>0)$ & The remaining time of staying current "off” state counting from $t_{s}$ \\
\hline$F_{\text {OFF }}\left(T^{0}\right)$ & The c.d.f. of $T^{0}$ \\
\hline$F_{O N}\left(T^{1}\right)$ & The c.d.f. of $T^{1}$ \\
\hline$\Gamma_{O F F}(\tilde{x}) / E\left\{T^{0}\right\}$ & The p.d.f. of $\tilde{x}$ remaining staying “off" [?]; $\Gamma_{\text {OFF }}(\tilde{x})=1-F_{\text {OFF }}(\tilde{x})$ \\
\hline$\Gamma_{O N}(\tilde{x}) / E\left\{T^{1}\right\}$ & The p.d.f. of $\tilde{x}$ remaining staying "on" [?]; $\Gamma_{\text {ON }}(\tilde{x})=1-F_{\text {ON }}(\tilde{x})$ \\
\hline$\tau_{d}(t),(d=0,1)$ & The average time of PU occupations on the channel during $\left(t_{s}, t_{s}+t\right)$ \\
\hline$\tilde{\tau}_{d}(t),(d=0,1)$ & The $\tau_{d}(t)$, when the state variation (on $\rightarrow$ off, or reversely) occurs at $t_{s}$ \\
\hline
\end{tabular}

The contamination of SU data delivery is mostly because of the suddenly returning of PU. When this case happens, the SNR (signal to noise ratio) or SINR (signal to interference plus noise ratio) would dramatically decrease. Thus, we introduce a threshold $L_{t h}$, which limits the average length of PU data during an indicated "off” spectrum sensing interval. Before going deep, we provide a metric $\tau_{0}($.$) which denotes the average length of the PU data during an indicated "off” spectrum sensing$ interval, as shown in Fig. 1. Fig. 1 is inspired by [6]. The variables in Fig. 1 are explained in Table. 1. $\tau_{0}($.$) can be written as Eq. 5, which is obtained by solving a group of integral equations with Laplace$ transform following that of [6].

$$
\tau_{0}(t)=\frac{\theta^{0}}{\theta^{1}+\theta^{0}} t-\frac{\theta^{0}}{\left(\theta^{1}+\theta^{0}\right)^{2}}\left(1-e^{-\left(\theta^{1}+\theta^{0}\right) t}\right)
$$

where the derivation Eq. 5 is omitted, which can be conceived as the "dual" problem of that in [6]. The interested reader may refer to it.

Define $P_{o}\left(\tau_{0}(\tau)>L_{t h}\right)$ denote the outage probability that the average length of the PU data during an "off” spectrum sensing interval $\tau$ is larger than threshold $L_{t h}$. Let $P_{o}<\rho_{o}$, where $\rho_{o}$ is a predefined constant, such that the retransmission is limited. Based on Eq. 5, $P_{o}\left(\tau_{0}(\tau)>L_{t h}\right)$ can be written as,

$$
P_{o}\left(\tau_{0}(\tau)>L_{t h}\right)=\frac{\int_{0}^{\tau-\Delta t_{t h}} f^{0}(t) d t}{\int_{0}^{\tau} f^{0}(t) d t}
$$

where at $\Delta t_{t h}, \tau_{0}\left(\Delta t_{t h}\right)=L_{t h}$. Eq. 6 is easy to understand. The numerator at the right side of Eq. 6 is the sum of the probabilities when $\tau_{0}($.$) is larger than L_{t h}$. The denominator at the right side of Eq. 6 performs the role of normalization. $f^{0}(t)$ is the distribution of the "off" periods. In the integral in Eq. 6, the probability distribution function (PDF) of $\tau_{0}(\cdot)$ is not Eq. 2, but $f^{0}(t)$. There are two reasons. The first one is that there is no state variation of PU from time 0 to time $t$ in the integral in Eq. 6. The other reason is that after this time $t$ the PU state begins to change, i.e., switch to "on". Please refer to the first pair of sub figures in Fig.1, for details. Eq. 2 is provided for comparison and explanation, thus Eq. 6 would become clear. Therefore, $D_{r e}\left(T_{p}, \tau\right)$ can be written as 


$$
T_{r e}\left(T_{p}, \tau\right)=\frac{T_{p}}{\tau}\left(P_{o} \cdot \tau\right)=T_{p} P_{o}
$$

where from Eq. 7 on, we have suppressed the parameters of $P_{o}\left(\tau_{0}(\tau)>L_{t h}\right)$ for convenience.

\section{The Optimal Solution}

According to Eq. 4 and Eq. 7, Eq. 3 can be written as

$$
\tau^{*}=\arg \min _{\tau}\left\{T_{p}\left(1+P_{o}\right) \frac{u \tau+t_{\text {win }}}{(1-u) \tau}\right\}
$$

The solution of Eq. 8 is discussed in this part. There is one implicit constraint for Eq. 8, i.e., $P_{o}<\rho_{o}$. We will discuss the $\tau^{*}$ according to the first derivative of Eq. 8 regarding the this implicit constraint. Since $\tau_{0}(t)$ is monotonically increasing with $t, P_{o}$ is also monotonically increasing with $\tau$. There is one special value during the varying of the spectrum sensing interval $\tau$, i.e., $\tau=\tau_{P_{o}}$, where at $\tau_{P_{o}}, P_{o}=\rho_{o}$. Recall, there is another special time point $\Delta_{t h}$ where $\tau_{0}\left(\Delta t_{t h}\right)=L_{t h}$. Obviously, $\tau^{*}$ can be obtained by investigating the relationship of $\Delta_{t h}$ and $\tau_{P_{o}}$.

Case $1 \Delta_{\text {th }} \leq \tau_{P_{o}}$. In this case, the set of the feasible values of $\tau$ are partitioned into two intervals, i.e., $\left(0, \Delta_{t h}\right]$ and $\left(\Delta_{t h}, \tau_{P_{o}}\right)$.

The derivative of Eq. 8 with $\tau \in\left(0, \Delta_{t h}\right]$ is, $-\frac{T_{P} t_{\text {win }}}{(1-u) \tau^{2}}<0$, thus, for $\in\left(0, \Delta_{t h}\right]$, when $\tau=\Delta_{t h}$, Eq. 8 is at its minimal value. Please note, for $\tau \in\left(0, \Delta_{t h}\right], P_{o}=0$.

The derivative of Eq. 8 with $\tau \in\left(\Delta_{t h}, \tau_{P_{o}}\right)$ is calculated, as shown in Eq. 9.

$$
T_{P}\{\underbrace{\frac{\partial P_{o}}{\partial \tau} \cdot \frac{u \tau+t_{\text {win }}}{(1-u) \tau}}_{A}+\underbrace{P_{o}\left(-\frac{t_{\text {win }}}{(1-u) \tau^{2}}\right)}_{B}\}
$$

where $\frac{\partial P_{o}}{\partial \tau}=\frac{\theta^{0}\left(e^{\theta^{0}\left(\tau-\Delta_{t h}\right)}-e^{-\theta^{0} \tau}\right)}{\left(1-e^{-\theta^{0} \tau}\right)^{2}}$. In Eq. 9, the relationship of term $A$ and term $B$ cannot be decided with the varying of $u, t_{\text {win }}$ and $\Delta_{t h}$. Therefore, the monotonicity of Eq. 8 cannot be decided for $\tau \in\left(\Delta_{t h}, \tau_{P_{o}}\right)$. We suggest a numerical search for the solution of Eq. 8 in $\left(\Delta_{t h}, \tau_{P_{o}}\right)$.

$\tau^{*}$ is the minimum one of the two solutions obtained above.

Case $2 \Delta_{t h}>\tau_{P_{o}}$. In this case, $\left(0, \Delta_{t h}\right] \supset\left(\tau_{P_{o}}, \Delta_{t h}\right)$, the problem of Eq. 8 becomes simple. Since $P_{o}=0$ for $\tau \in\left(0, \Delta_{t h}\right]$, thus, according to the analysis in Case $1, \tau^{*}=\Delta_{t h}$.

Remark 1: In the erroneous spectrum sensing case, $P_{f} \neq 0$ and $P_{m} \neq 0$. The (discovered) busy portion of the PU channel, which is denoted by $u$ in the error-free case. is redenoted as $\tilde{u}$, $\tilde{u}=\left(1-P_{f}\right) u+P_{m} \cdot(1-u)$ in the erroneous case. On the other hand, the corresponding (discovered) useful portion of the PU channel, which is denoted as $\tilde{\alpha}$, can be written as $\tilde{\alpha}=\left(1-P_{m}\right)(1-u) \frac{\tau}{t_{\text {win }}+\tau}+P_{f} \cdot u \frac{\tau}{t_{\text {win }}+\tau}$. Thus, the delay minimization problem in this paper can be reformulated. Due to its similarity, no more is explained.

\section{Numerical Results}


The numerical values of $\tau$ in Eq. 8 is plotted with delay in Fig.2. The parameter setting is as follows. $T_{P}=10 \mathrm{~s} . t_{\text {win }}=0.1 . \theta^{1}=\theta^{0}=1$, hence $u=0.5 . L_{t h}=0.1 \cdot \rho_{o}=0.2$. The values of $\Delta_{t h}$ and $\tau_{P_{o}}$ are calculated, i.e., $\Delta_{t h}=0.5249<\tau_{P_{o}}=0.6221$. As shown in Fig.2, the dash line denotes the first interval $\tau \in\left(0, \Delta_{t h}\right]$, while the solid line denotes the second interval $\tau \in\left(\Delta_{t h}, \tau_{P_{o}}\right)$. The star markers resides in the plotted curve in Fig. 2 is the optimal value $\tau^{*}$.

Table 2 The Optimal Spectrum Sensing Interval with Different Channel Parameters (unit sec..).

\begin{tabular}{|c|l|l|l|l|l|}
\hline$E\left[T^{1}\right]$ & 1.50 & 0.50 & 1.00 & 3.00 & 3.00 \\
\hline$E\left[T^{0}\right]$ & 0.80 & 2.50 & 1.00 & 2.50 & 3.00 \\
\hline$\tau^{*}$ & 0.46 & 0.98 & 0.52 & 0.77 & 0.85 \\
\hline
\end{tabular}

We have tested more cases of different channel conditions. The results are shown in Table. 2. The values of $E\left[T^{0}\right]$ and $E\left[T^{1}\right]$ are borrowed from [6]. The other parameters are the same as these in Fig.2.

To show the improvement in delay induced by delivering an SU packet $T_{P}$, the proposed method is compared to the method of [6]. The results are plotted in Fig.3. The x-axis of Fig.3 is $E\left[T^{0}\right] / E\left[T^{1}\right]$, where $E\left[T^{1}\right]$ is set to 2s and $E\left[T^{0}\right]$ varies. The other parameters are the same as those of Fig.2. As shown by Fig.3, the delay of the proposed method is reduced compared to that of [6].

\section{Conclusion}

In this work, we have studied the delay minimization problem regarding the varying of spectrum sensing interval for CR. Especially, the retransmission of SU data is considered. The optimal solution is obtained. The procedures of finding the optimal solution can be simplified for certain parameters. Our results would inspire further study about the throughput enhancement of cognitive radio, particularly those considering the retransmission.

\section{Acknowledgements}

This research was supported in part by National Science Foundation of China (No.61201180), Beijing Municipal Natural Science Foundation (N0.4132055), and Excellent Young Scholars Research Fund of Beijing Institute of Technology.

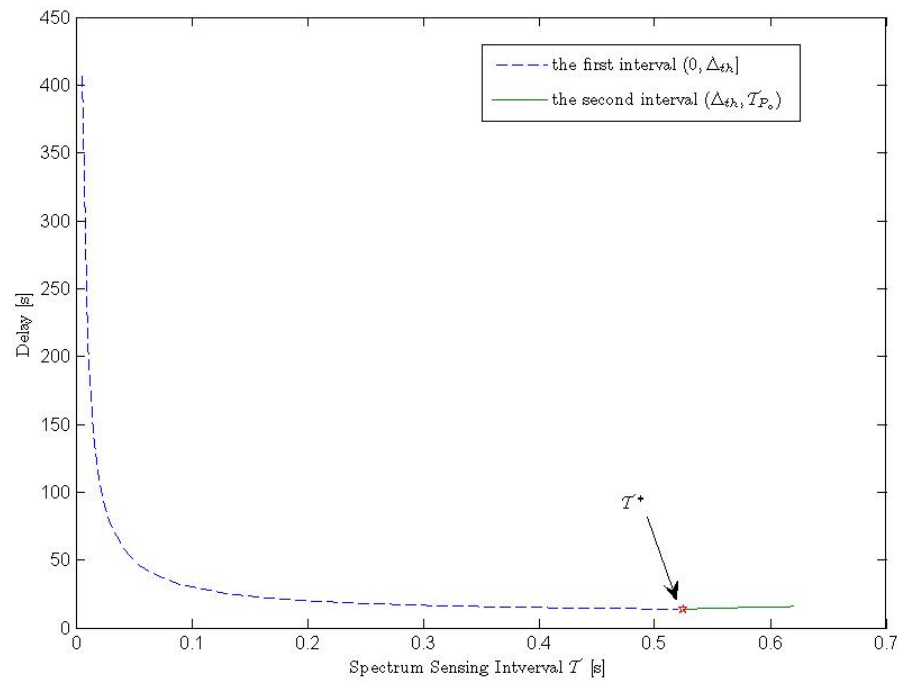

Fig. 2. The delay induced by delivering an SU packet $T_{P}$ on a PU channel with spectrum sensing interval $\tau$. The star marker in the curve indicates the optimal value $\tau=\tau^{*}$. 


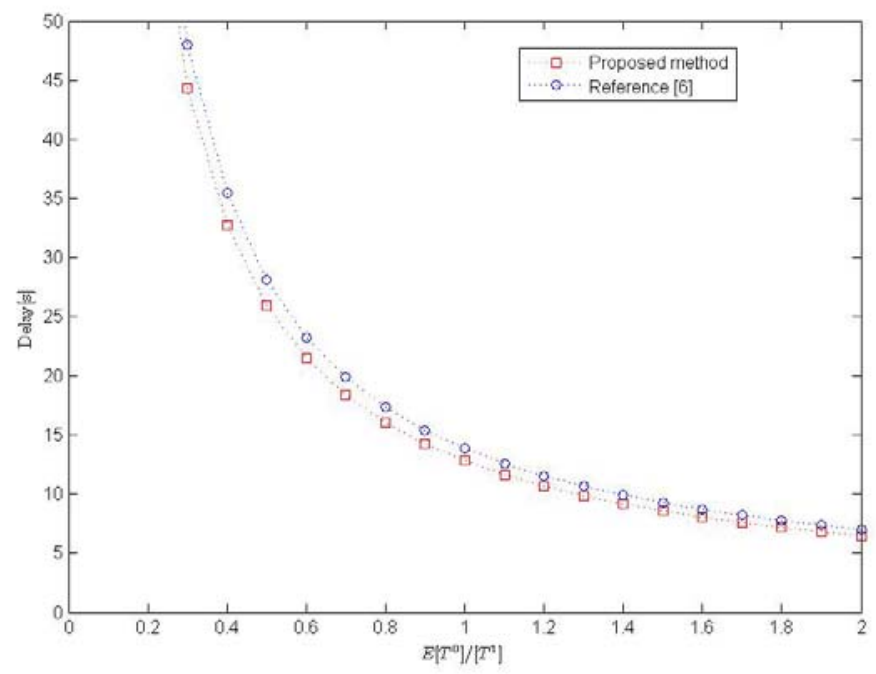

Fig. 3. The delay induced by delivering an SU packet $T$, when the spectrum sensing interval is set according to our proposed method and that of [6], respectively.

\section{References}

[1] S. Haykin, Cognitive radio: brain-empowered wireless communications, IEEE J. Sel. Areas Commun., vol. 23, no. 2, pp. 201-220, Feb. 2005.

[2] Yucek, T., and Arslan, H., A survey of spectrum sensing algorithms for cognitive radio applications, IEEECommunications Surveys \& Tutorials, vol.11, no.1, pp. 116-130, 2009.

[3] A.O. Ercan, M.O. Sunay, Energy Sensing Strategy Optimization for Opportunistic Spectrum Access, IEEE Commun. Letters, vol.16, no.6, pp.828-830, June 2012.

[4] H. Tang, Some physical layer issues of wide-band cognitive radio systems, in Proc. IEEE Int. Symposium on New Frontiers in Dynamic Spectrum Access Networks, Baltimore, Maryland, USA, Nov. 2005, pp. 151-159.

[5] Josep Font-Segura, Xiaodong Wang, GLRT-Based Spectrum Sensing for Cognitive Radio with Prior Information IEEE Trans. Commun., vol.58, no.7, pp.2137-2146, July 2010.

[6] H. Kim and K. G. Shin, Efficient discovery of spectrum opportunities with MAC-layer sensing in cognitive radio networks, IEEE Trans. Mobile Comput., vol. 7, no. 5, pp. 533-545, May 2008.

[7] Anh Hoang, Ying-Chang Liang, Yonghong Zeng, Adaptive joint scheduling of spectrum sensing and data transmission in cognitive radio networks, IEEE Trans Commun., vol.58, no.1, pp.235-246, Jan. 2010.

[8] L. Yang, L. Cao, and H. Zheng, Proactive channel access in dynamic spectrum networks, Physical Communication, vol. 1, pp. 103-111, 2008.

[9] Hai Jiang, Lifeng Lai, Rongfei Fan and Poor H. V., Optimal selection of channel sensing order in cognitive radio, IEEE Trans. Wireless Commun., vol. 8, no. 1, pp. 297-307, Jan. 2009.

[10] Rong-Rong Chen and Xin Liu, Delay performance of threshold policy in dynamic spectrum access, IEEE Trans. Wireless Commun., vol. 10, no. 7, pp. 2283-2293, Jul. 2011. 